\title{
Comparative Antimicrobial Activities of Linezolid and Vancomycin against Gram-Positive Clinical Isolates from Hospitals in Kuwait
}

\author{
O.A. Phillips ${ }^{a}$ E.E. Udo ${ }^{b}$ A.A.M. Alic E.M. Mokaddas ${ }^{b}$ \\ aDepartment of Pharmaceutical Chemistry, Faculty of Pharmacy, ${ }^{b}$ Department of Microbiology, Faculty of Medicine, \\ and ${ }^{\mathrm{c}}$ Department of Chemistry, Faculty of Science, Kuwait University, Kuwait
}

\section{Key Words}

Antimicrobial agents · Resistance - Gram-positive bacteria $\cdot$ Linezolid $\cdot$ Oxazolidinone $\cdot$ Vancomycin

\begin{abstract}
Objectives: The increasing prevalence of resistant grampositive bacteria, emergence of vancomycin-resistant enterococci and the isolation of vancomycin-intermediate resistant Staphylococcus aureus have emphasized the need for new antimicrobial agents to treat infections caused by these organisms. We studied the comparative antimicrobial activities of linezolid, a new and totally synthetic oxazolidinone antibacterial agent and vancomycin against resistant gram-positive clinical isolates from four hospitals in Kuwait. Methods: The organisms were isolated from clinical samples including throat and wound swabs, urine and blood, and identified by cultural and biochemical characteristics. The minimum inhibitory concentration (MIC) of linezolid and vancomycin against S. aureus, coagulase-negative staphylococci, Streptococcus pneumoniae and enterococci was determined by the agar dilution method. Results: Linezolid demonstrated MIC ranges of $0.5-4 \mathrm{mg} / \mathrm{l}$ against methicillinresistant $S$. aureus (MRSA) and methicillin-susceptible $S$. aureus, 1-4 mg/l against methicillin-resistant coagulasenegative staphylococci (MR-CNS) and methicillin-sensi-
\end{abstract}

\section{KARGER}

Fax +41613061234

E-Mail karger@karger.ch

www.karger.com

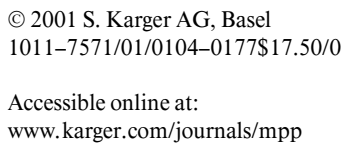

tive coagulase-negative staphylococcal strains, and a narrow range of $0.5-1 \mathrm{mg} / \mathrm{l}$ against penicillin-resistant $S$. pneumoniae (PRSP). The range of vancomycin MICs against MRSA, MR-CNS and PRSP was 0.5-4, 0.5-1 and $0.25-0.5 \mathrm{mg} / \mathrm{l}$, respectively. The MIC values of linezolid and vancomycin against vancomycin-susceptible enterococci were 1-2 and 0.5-4 mg/l, respectively. However, linezolid demonstrated a lower MIC of $2 \mathrm{mg} / \mathrm{l}$ against vancomycin-intermediate resistant enterococci and vancomycin-resistant enterococci than vancomycin (MICs of 8 and $>512 \mathrm{mg} / \mathrm{l}$, respectively). Conclusion: The results indicated that linezolid demonstrated activity comparable to vancomycin against all staphylococcal, vancomycin-susceptible enterococci and PRSP strains, but demonstrated superior activity against vancomycin-intermediate resistant enterococci and vancomycin-resistant enterococci. In addition, linezolid was highly effective against all multidrug-resistant gram-positive clinical isolates from hospitals in Kuwait.

Copyright $\odot 2001$ S. Karger AG, Basel

\section{Introduction}

The emergence of multidrug-resistant gram-positive bacterial pathogens including Staphylococcus aureus, Staphylococcus epidermidis, Streptococcus pneumoniae,
Dr. O.A. Phillips

Faculty of Pharmacy, Kuwait University

PO Box 24923

13110 Safat (Kuwait)

Tel. +965 531 2300, Ext. 6048, Fax +965 534 2807, E-Mail dphillips@hsc.kuniv.edu.kw 
Enterococcus faecium and Enterococcus faecalis has resulted in significant challenges to effective therapy of diseases caused by these pathogens $[1,2]$. These multidrugresistant gram-positive pathogens have become a serious problem in hospital and community settings, particularly in cases where treatment with glycopeptides has failed [3]. Parenteral vancomycin is the only major treatment option in many cases of methicillin-resistant $S$. aureus (MRSA) infections. However, the emergence of $S$. aureus strains with reduced suscpetibility to vancomycin in Japan, France and the United States is alarming, and may limit the efficacy and use of this agent in the health care community [4-8]. Linezolid (Zyvox ${ }^{\mathrm{TM}}$ ) is the first member of a structurally unique class of antimicrobial agents referred to as the oxazolidinones, approved by the United States Food and Drug Administration in April 2000, for the treatment of multidrug-resistant gram-positive bacterial infections [9]. Importantly, multidrug-resistant organisms such as methicillin-resistant staphylococci, and those with reduced susceptibility to vancomycin (minimum inhibitory concentration, MIC, $8 \mathrm{mg} / \mathrm{l}$ ) [9], penicillin- and macrolide-resistant pneumococci and vancomycin-resistant enterococci (VRE), appear to be fully susceptible to linezolid $[10,11]$. Controlled, randomized clinical studies have documented the efficacy and safety of linezolid in treatment of hospital- and community-acquired pneumonia, uncomplicated and complicated skin and soft tissue infections and infections caused by VRE [10, 11]. Linezolid has almost 100\% bioavailability, with the area under the plasma concentration curve being identical after oral or intravenous (i.v.) administration. This enables initial oral administration of linezolid in those patients who can absorb the drug normally and also enables an early step-down therapy from i.v. to oral dosing $[11,12]$. Oxazolidinones act primarily against grampositive bacteria by inhibiting protein synthesis: binding sites have been found on both $30 \mathrm{~S}$ and $50 \mathrm{~S}$ ribosomal subunits, rRNA being the only target, although the involvement of $16 \mathrm{~S}$ and $23 \mathrm{~S}$ rRNA binding has been suggested [13].

The activity of linezolid has been evaluated against gram-positive cocci isolated from hospitals in the UK [14] and North America $[15,16]$. However, no such study has been conducted on clinical isolates from Kuwait and the Gulf region. Therefore, as part of our ongoing efforts to monitor incidence of multidrug-resistant gram-positive pathogens from Kuwait hospitals, we initiated this study to evaluate the comparative antimicrobial activities of linezolid and vancomycin against gram-positive clinical isolates from four hospitals in Kuwait.
Table 1. Comparison of in vitro activity of linezolid and vancomycin against clinical isolates

\begin{tabular}{llll}
\hline Bacteria & $\begin{array}{l}\text { Isolates } \\
\text { tested }\end{array}$ & \multicolumn{2}{l}{ MIC (mg/l) range for } \\
\cline { 3 - 4 } & & linezolid & vancomycin \\
\hline MSSA & 10 & $2-4$ & 1 \\
MRSA & 40 & $0.5-4$ & $0.5-4$ \\
MS-CNS & 10 & $1-4$ & $0.5-1$ \\
MR-CNS & 40 & $1-4$ & $0.5-1$ \\
PRSP & 21 & $0.5-1$ & $0.25-0.5$ \\
VSE & 20 & $1-2$ & $0.5-4$ \\
VIRE & 5 & 2 & 8 \\
VRE & 5 & 2 & $\geq 512$ \\
\hline
\end{tabular}

\section{Materials and Methods}

\section{Bacterial Isolates}

A total of 151 clinical isolates of gram-positive pathogenic bacteria, collected between 1994 and 1999 from four hospital (Al-Mubarak, Adan, Amiri and Ibn Sina) in Kuwait, were utilized for this study. The organisms were isolated from different clinical samples including throat and wound swabs, urine and blood. Isolates consisted of 40 MRSA, 10 methicillin-susceptible $S$. aureus (MSSA), 40 methicillin-resistant coagulase-negative staphylococci (MR-CNS), 10 methicillin-sensitive coagulase-negative staphylococci (MS-CNS), 21 penicillin-resistant $S$. pneumoniae (PRSP), and 30 enterococcal strains. The enterococcal strains consisted of 20 vancomycin-susceptible enterococci (VSE), 5 vancomycin-intermediate resistant enterococci (VIRE), and 5 VRE. The isolates were stored at $-80^{\circ} \mathrm{C}$ in $10 \%$ (w/v) skim milk until required. The $S$. aureus strains were selected based on their resistance phenotypes and patterns generated after pulse field gel electrophoresis. Coagulase-negative staphylococcal strains were selected based on differences in their resistance phenotypes.

The enterococcal and streptococcal strains were identified based on cultural characteristics, results of catalase and coagulase tests, optochin sensitivity, bile esculin and biochemical profiles using API staph and API strept (bioMérieux, Marcy-l'Etole, France).

\section{Antibiotic Susceptibility Testing}

Minimum Inhibitory Concentration. The MIC of linezolid and vancomycin against $S$. aureus, coagulase-negative staphylococci and enterococci was determined by the agar dilution method as described previously [17]. Linezolid (Zyvox) was provided by Pharmacia Corp. (Kalamazoo, Mich., USA) while vancomycin was purchased from Sigma Chemical Company (St. Louis, Mo., USA). The MICs of linezolid against $S$. pneumoniae were determined by the agar dilution method on Mueller-Hinton agar supplemented with $5 \% \mathrm{v} / \mathrm{v}$ sheep blood. The MIC of vancomycin against $S$. pneumoniae was determined with E-test strips (AB Biodisk, Solna, Sweden). Linezolid solution was prepared according to the method provided by the manufacturer (Pharmacia Corp.). Bacterial suspensions in tryptic soy broth were distributed in wells of a multipoint inoculator, which was used to spot approximately $10^{6} \mathrm{CFU}$ per spot onto Mueller-Hinton agar plates containing dilutions of antibiotics ranging from 0.25 to 
Table 2. MIC distribution of linezolid and vancomycin against clinical isolates

\begin{tabular}{|c|c|c|c|c|c|c|c|c|c|c|}
\hline \multirow[t]{3}{*}{ Organisms } & \multicolumn{10}{|c|}{ Percent of isolate with specified MICs (mg/l) for } \\
\hline & \multicolumn{4}{|c|}{ linezolid, mg/l } & \multicolumn{6}{|c|}{ vancomycin, $\mathrm{mg} / \mathrm{l}$} \\
\hline & 0.5 & 1.0 & 2.0 & 4.0 & $(0.25) 0.5$ & 1.0 & 2.0 & 4.0 & 8 & $\geq 512^{\mathrm{a}}$ \\
\hline MRSA $(n=40)$ & 2.5 & 12.5 & 42.5 & 42.5 & 2.5 & 25 & 40 & 32.5 & - & - \\
\hline $\operatorname{MSSA}(n=10)$ & - & - & 10 & 90 & - & 100 & - & - & - & - \\
\hline MR-CNS $(n=40)$ & - & 82.5 & 12.5 & 5 & 7.5 & 92.5 & - & - & - & - \\
\hline MS-CNS $(n=10)$ & - & 80 & - & 20 & 10 & 90 & - & - & - & - \\
\hline $\operatorname{PRSP}(\mathrm{n}=21)$ & 19 & 81 & - & - & (9.5) 90.5 & - & - & - & - & - \\
\hline $\operatorname{VSE}(n=20)$ & - & 60 & 40 & - & 10 & 70 & 15 & 5 & - & - \\
\hline $\operatorname{VIRE}(\mathrm{n}=5)$ & - & - & 100 & - & - & - & - & - & 100 & - \\
\hline $\operatorname{VRE}(\mathrm{n}=5)$ & - & - & 100 & - & - & - & - & - & - & 100 \\
\hline
\end{tabular}

a One VRE strain showed MIC of 1,024 mg/l.

$512 \mathrm{mg} / \mathrm{l}$. The plates were incubated at $35^{\circ} \mathrm{C}$ for $24 \mathrm{~h}$. The MIC was defined as the lowest concentration of antibiotic that completely inhibited growth of bacteria after 24-hour incubation at $35^{\circ} \mathrm{C}$.

Disk Diffusion Susceptibility Testing. The isolates were also tested for susceptibility to other antibacterial agents by the disk diffusion method. Methicillin resistance was detected on Mueller-Hinton agar supplemented with $2 \% \mathrm{NaCl}$ and incubated at $35^{\circ} \mathrm{C}$ for $24 \mathrm{~h}$. Susceptibility testing for $S$. pneumoniae was performed on Mueller-Hinton agar supplemented with $5 \%$ sheep blood. Penicillin resistance in $S$. pneumoniae was screened with oxacillin disks $(1 \mu \mathrm{g})$ and confirmed by determining MICs to penicillin with E-test strips (AB Biodisk). High-level resistance to gentamicin in enterococci was tested with disks containing $120 \mu \mathrm{g}$ of gentamicin.

\section{Results}

\section{Susceptibility of Linezolid Vancomycin}

The 151 gram-positive clinical isolates were evaluated for their comparative susceptibilities to linezolid and vancomycin. The MIC values are summarized in tables 1 and 2. Both antibiotics demonstrated comparable antimicrobial activity against both the sensitive and resistant clinical isolates of staphylococci. The MIC ranges of 1-4 and $0.5-1 \mathrm{mg} / 1$ were observed for linezolid and vancomycin, respectively, against all MR-CNS strains tested. Eightythree percent and $93 \%$ of the MR-CNS showed comparable MICs of $1 \mathrm{mg} / \mathrm{ml}$ for linezolid and vancomycin, respectively. With linezolid, $15 \%$ of the MRSA strains showed MIC ranges of $0.5-1 \mathrm{mg} / 1$, while more than $80 \%$ of the MRSA strains demonstrated MIC ranges of 2$4 \mathrm{mg} / \mathrm{l}$. Vancomycin demonstrated an MIC range of 0.5$4 \mathrm{mg} / \mathrm{l}$ against MRSA, with a total of about 27 and $73 \%$ falling within the MIC ranges of $0.5-1$ and $2-4 \mathrm{mg} / \mathrm{l}$, respectively. All PRSP isolates were equally susceptible to vancomycin and linezolid with MICs in the narrow ranges of $0.25-0.5$ and $0.5-1 \mathrm{mg} / 1$, respectively.

Linezolid was found to be highly effective against all clinical isolates of enterococci, with MICs in the range of 1-2 mg/l, while the MICs of vancomycin against VIRE $(8 \mathrm{mg} / \mathrm{l})$ and VRE $(\geq 512 \mathrm{mg} / \mathrm{l})$ were found to be much higher.

\section{Resistance to Antimicrobial Agents}

A total of 151 gram-positive clinical isolates consisting of 40 MRSA, 10 MSSA, 40 MR-CNS, 10 MS-CNS, 20 VSE, 5 VIRE, 5 VRE and 21 PRSP were evaluated for their susceptibilities to a number of selected antibiotics including ciprofloxacin, vancomycin, teicoplanin and $\beta$ lactams. The isolates were selected to reflect a wide array of susceptibility patterns. The results of testing the antimicrobial activities of different antibiotics against the clinical isolates indicated that all of the MRSA isolates were resistant to penicillin G. However, 95, 85 and 80\% of them were resistant to gentamicin and tetracycline, erythromycin and ciprofloxacin, and trimethoprim, respectively, but were fully susceptible to both teicoplanin and vancomycin. The MSSA isolates were all susceptible to gentamicin, ciprofloxacin and vancomycin, but showed high resistance (rate of about $80 \%$ ) to penicillin G. Twenty percent of the MSSA isolates were resistant to both tetracycline and trimethoprim.

The majority of the MR-CNS isolates demonstrated varying levels of resistance to most of the antimicrobial 
agents evaluated, including teicoplanin, ciprofloxacin, penicillin $\mathrm{G}$, trimethoprim, tetracycline and gentamicin. However, resistance to vancomycin was not detected. Antibiotic resistance among the MS-CNS clinical isolates was generally low. However, resistance to penicillin $G$, gentamicin, trimethoprim and chloramphenicol was observed, while the MS-CNS strains were susceptible to erythromycin, clindamycin, tetracycline, ciprofloxacin, teicoplanin and vancomycin.

Among the PRSP isolates, resistance rates of between 15 and $80 \%$ were observed against most of the other antimicrobial agents evaluated, including ceftriaxone, cefotaxime, cefuroxime, trimethoprim, erythromycin and tetracycline. However, all of the $S$. pneumoniae strains were susceptible to vancomycin.

The enterococcal clinical isolates were found to be multidrug-resistant towards the various antibiotics evaluated, including ampicillin, chloramphenicol, ciprofloxacin, erythromycin, gentamicin, teicoplanin, tetracycline and vancomycin. About 66.6, 16.7 and $16.7 \%$ of enterococcal clinical isolates were found to be VSE, VIRE (MIC 8-32 mg/l), and VRE (MIC > $32 \mathrm{mg} / \mathrm{l}$ ) strains, respective$1 \mathrm{y}$.

\section{Discussion}

Antimicrobial resistance continues to pose serious problems worldwide with significant impacts on therapeutic strategies for a variety of bacterial, fungal and protozoal infections [18]. The emergence of bacterial resistance to a variety of antimicrobial agents such as the $\beta$ lactams, aminoglycosides, macrolides, glycopeptides and quinolones among gram-positive bacteria (including staphylococci, enterococci and streptococci) is of much concern because of the lack of effective alternative agents [19]. The continued increase in bacterial infections caused by MRSA, VRE and PRSP is causing significant therapeutic problems. Parenteral vancomycin has been the only reliable treatment option in many cases of serious MRSA infections [20]. However, the recently reported cases of $S$. aureus with intermediate resistance to vancomycin (MIC $8 \mathrm{mg} / \mathrm{l}$ ) [9], first from Japan and then from the United States, have given cause for alarm in the health care community [7]. This situation warranted the urgent search for new, safe and reliable agents for the treatment of infections caused by multidrug-resistant gram-positive pathogens. Therefore, the development and subsequent approval of linezolid for clinical use against gram-positive pathogens is a welcome development.
In the present study, the activity of linezolid was evaluated in comparison to that of vancomycin against selected gram-positive pathogenic bacteria isolated from various infection sites in patients from Kuwait hospitals. All the $S$. aureus isolates were susceptible to linezolid and vancomycin with MIC ranges of $0.5-4$ and $0.5-4 \mathrm{mg} / 1$, respectively (tables 1,2 ); these strains were also susceptible to teicoplanin. However, susceptibility testing results indicated that most of the MRSA isolates were also multidrugresistant, including high resistance rates for trimethropin and ciprofloxacin. This underlines the need for a new and effective agent against these isolates. The coagulase-negative staphylococci were also susceptible to linezolid with an MIC range of 1-4 mg/l. As observed for the MRSA isolates, the MR-CNS strains were also resistant to the other antibiotics tested. In general, higher resistance rates to most of the antibiotics were seen amongst MRSA and MR-CNS than amongst MSSA and MS-CNS, a result similar to observations by Henwood et al. [14] on clinical isolates from UK hospitals. Although high resistance rates to most of the antibiotics including cefotaxime (29\%), ceftriaxone $(52 \%)$ and cefuroxime (67\%) were observed for PRSP, they were all susceptible to ciprofloxacin and vancomycin and all were inhibited by $0.5-1$ and $0.25-$ $0.5 \mathrm{mg} / \mathrm{l}$ of linezolid and vancomycin, respectively (tables 1,2$)$. The PRSP clinical isolates from our study were susceptible to ciprofloxacin. However, a resistance rate of $1.4 \%$ for ciprofloxacin against $S$. pneumoniae and a documentation of a significant increase in antimicrobial resistance with $S$. pneumoniae in the USA over a period of 5 years was recently reported by Doern et al. [15].

The prevalence of ciprofloxacin and erythromycin resistance was found to be high among the enterococcal isolates. However, all VSE isolates were inhibited by 1-2 and $0.5-4 \mathrm{mg} / \mathrm{l}$ of linezolid and vancomycin, respectively (tables 1, 2). Linezolid inhibited strains of VIRE and multidrug-resistant VRE in vitro at a concentration of $2 \mathrm{mg} / \mathrm{l}$, compared to MIC values of 8 and $\geq 512 \mathrm{mg} / \mathrm{l}$ with vancomycin (tables 1, 2).

Overall, the antibacterial activity of linezolid was comparable to that of vancomycin against sensitive and resistant isolates of staphylococci, S. pneumoniae, and VSE from patients in Kuwait hospitals. These results are in agreement with results of other studies that have evaluated the effectiveness of linezolid against resistant grampositive clinical isolates from other countries [14, 21-23]. Linezolid is a promising new agent active against grampositive clinical isolates, which can serve as a viable alternative in the treatment of serious gram-positive infections that are becoming increasingly intractable. 


\section{Conclusion}

The antimicrobial activity of linezolid against multidrug-resistant gram-positive bacteria including MRSA, MR-CNS, PRSP, and VSE was comparable to that of vancomycin. However, linezolid clearly demonstrated activity superior to vancomycin against VIRE and VRE strains, thus signifying its potential usefulness in the treatment of infections due to multidrug-resistant gram-positive cocci.

\section{Acknowledgment}

Supported by Kuwait University grant numbers PC 01/00 and MI 01-00.

\section{References}

1 Plouffe JF: Emerging therapies for serious Gram-positive bacterial infections: A focus on linezolid. Clin Infect Dis 2000;31(suppl 4):S144-S149.

2 Ford C, Hamel J, Stapert D, Moerman J, Hutchinson D, Barbachyn M, Zurenko G: Oxazolidinones: A new class of antimicrobials. Infect Med 1999; 16:435-445.

3 Shetty N, Wilson AP: Sitafloxacin in the treatment of patients with infections caused by vancomycin-resistant enterococci and methicillinresistant Staphylococcus aureus. J Antimicrob Chemother 2000;46:633-638.

4 Smith TL, Pearson ML, Wilcox KR, Cruz C, Lancaster MV, Robinson-Dunn B, Tenover FC, Zervos MJ, Band JD, White E, Jarvis WR: Emergence of vancomycin resistance in Staphylococcus aureus. Glycopeptide-Intermediate Staphylococcus aureus Working Group. N Engl J Med 1999;340:493-501.

5 Inaba Y, Hiramatsu K: Emergence of heteroVISA strains in Japanese hospitals and its countermeasure. Nippon Rinsho 1998;56: 2699-2705.

6 Hiramatsu K, Hanaki $\mathrm{H}$, Ino T, Yabuta K, Oguri T, Tenover FC: Methicillin-resistant Staphylococcus aureus clinical strain with reduced vancomycin susceptibility. J Antimicrob Chemother 1997;40:135-136.

7 Beavers N: Doomsday bugs? Fighting antibiotic resistance. Drug Top 1998;4:47-54.

8 Ploy MC, Grelaud C, Martin C, de Lumley L, Denis F: First clinical isolate of vancomycinintermediate Staphylococcus aureus in a French hospital. Lancet 1998;351:1212.
9 Chien JW, Kucia ML, Salata RA: Use of linezolid, an oxazolidinone, in the treatment of multidrug-resistant gram-positive bacterial infections. Clin Infect Dis 2000;30:146-151.

10 Karchmer AW: Nosocomial bloodstream infections: Organisms, risk factors, and implications. Clin Infect Dis 2000;31(suppl 4):S139S143.

11 Norrby R: Linezolid: A review of the first oxazolidinone. Expert Opin Pharmacother 2001;2: 293-302.

12 Bain KT, Wittbrodt ET: Linezolid for the treatment of resistant gram-positive cocci. Ann Pharmacother 2001;35:566-575.

13 Matassova NB, Rodnina MV, Endermann R, Kroll HP, Pleiss U, Wild H, Wintermeyer W: Ribosomal RNA is the target of oxazolidinones, a novel class of transitional inhibitors. RNA 1999:5:939-946.

14 Henwood CJ, Livermore DM, Johnson AP, James D, Warner M, Gardiner A: Susceptibility of Gram-positive cocci from 25 UK hospitals to antimicrobial agents including linezolid. Linezolid Study Group. J Antimicrob Chemother 2000;46:931-940.

15 Doern GV, Heilmann KP, Huynh HK, Rhomberg PR, Coffman SL, Brueggemann AB: Antimicrobial resistance among clinical isolates of Streptococcus pneumoniae in the United States during 1999-2000, including a comparison of resistance rates since 1994-1995. Antimicrob Agents Chemother 2001;45:1721-1729.
16 Pelton SI, Figueira M, Albut R, Stalker D: Efficacy of linezolid in experimental otitis media. Antimicrob Agents Chemother 2000;44:654657.

17 National Committee for Clinical Laboratory Standards. Performance standards for antimicrobial disk susceptibility tests. Wayne, PA, NCCLS, (1998).

18 Chu DT, Plattner JJ, Katz L: New directions in antibacterial research. J Med Chem 1996;39: 3853-3874.

19 Gold HS, Moellering RC Jr: Antimicrobialdrug resistance. N Engl J Med 1996;335:14451453.

20 Brighty KE, Kohlbrenner W, McGuirk PR: Recent developments in antibacterial resistance mechanisms. Annu Rep Med Chem 1993;28: 141-150.

21 Patel R, Rouse MS, Piper KE, Steckelberg JM: In vitro activity of linezolid against vancomycin-resistant enterococci, methicillin-resistant Staphylococcus aureus and penicillin-resistant Streptococcus pneumoniae. Diagn Microbiol Infect Dis 1999;34:119-122.

22 Lizondo J, Rabasseda X, Castaner J: Linezolid Drugs Future 1996;21:1116-1123.

23 Noskin GA, Siddiqui F, Stosor V, Hacek D, Peterson LR: In vitro activities of linezolid against important Gram-positive bacterial pathogens including vancomycin-resistant enterococci. Antimicrob Agents Chemother 1999;43:2059-2062. 